\title{
LABORATORY PHOTO-CHEMISTRY OF PAHs: IONIZATION VERSUS FRAGMENTATION
}

\author{
Junfeng Zhen $^{1,2,3,4}$, Pablo Castellanos ${ }^{1,2}$, Daniel M. PaArdekooper ${ }^{2}$, Niels Ligterink $^{1,2}$, Harold Linnartz $^{2}$, \\ Laurent Nahon $^{5}$, Christine Joblin ${ }^{3,4}$, and Alexander G. G. M. Tielens ${ }^{1}$ \\ ${ }^{1}$ Leiden Observatory, University of Leiden, P.O. Box 9513, NL-2300 RA Leiden, The Netherlands; zhen@strw.leidenuniv.nl, junfeng.zhen@irap.omp.eu \\ ${ }^{2}$ Sackler Laboratory for Astrophysics, Leiden Observatory, University of Leiden, P.O. Box 9513, NL-2300 RA Leiden, The Netherlands \\ Universitè de Toulouse, UPS-OMP, IRAP, Toulouse, France \\ ${ }_{5}^{4}$ CNRS, IRAP, 9 Av. colonel Roche, BP 44346, F-31028 Toulouse Cedex 4, France \\ ${ }^{5}$ Synchrotron SOLEIL, L'Orme des Merisiers, F-91192 Gif sur Yvette Cedex, France \\ Received 2015 March 19; accepted 2015 April 6; published 2015 April 23
}

\begin{abstract}
Interstellar polycyclic aromatic hydrocarbons (PAHs) are expected to be strongly processed by vacuum ultraviolet photons. Here, we report experimental studies on the ionization and fragmentation of coronene $\left(\mathrm{C}_{24} \mathrm{H}_{12}\right)$, ovalene $\left(\mathrm{C}_{32} \mathrm{H}_{14}\right)$ and hexa-peri-hexabenzocoronene $\left(\mathrm{HBC} ; \mathrm{C}_{42} \mathrm{H}_{18}\right)$ cations by exposure to synchrotron radiation in the range of $8-40 \mathrm{eV}$. The results show that for small PAH cations such as coronene, fragmentation (H-loss) is more important than ionization. However, as the size increases, ionization becomes more and more important and for the HBC cation, ionization dominates. These results are discussed and it is concluded that, for large PAHs, fragmentation only becomes important when the photon energy has reached the highest ionization potential accessible. This implies that PAHs are even more photo-stable than previously thought. The implications of this experimental study for the photo-chemical evolution of PAHs in the interstellar medium are briefly discussed.
\end{abstract}

Key words: astrochemistry - ISM: molecules - methods: laboratory: molecular - molecular processes ultraviolet: ISM

\section{INTRODUCTION}

Strong emission features at 3.3, 6.2, 7.7, 8.6, and $11.2 \mu \mathrm{m}$ dominate the infrared (IR) spectra of the interstellar medium (ISM) of the Milky Way as well as galaxies in the local and far universe to redshift of $\sim 3$ (Sellgren 1984; Allamandola et al. 1989; Puget \& Leger 1989; Genzel et al. 1998; Armus et al. 2007; Lutz et al. 2007; Tielens 2013). These features are generally attributed to IR fluorescence of large $(\sim 50 \mathrm{C}$-atom) polycyclic aromatic hydrocarbon $(\mathrm{PAH})$ molecules pumped by UV photons. These species must be abundant, ubiquitous, and contain $\sim 10 \%$ of the elemental carbon and they play an important role in the ionization and energy balance of the ISM of galaxies (Tielens 2008 and references therein). The astronomical PAH population is expected to exist in different charge and (de)hydrogenation states depending on the environment. As the IR spectral characteristics of PAHs are sensitive to the charge and (de)hydrogenation state, the observed spectrum of IR emission features will reflect the local physical conditions, and thereby offer probes of the environment of the emitting species (Tielens 2005). In the ISM, PAH molecules are necessarily exposed to continuous broadband UV radiation, and a large fraction of PAHs are expected to be singly, doubly, or even triply ionized. Indeed, models have predicted that for typical electron density and radiation field intensity values in photodissociation regions, the population of PAHs ${ }^{++}$may well exceed that of $\mathrm{PAHs}^{+}$(Bakes et al. 2001a, 2001b). The possible presence of multiply charged PAH ions in the ISM - first proposed by Leach (1986) - has received renewed interest with their potential involvement in the extended red emission (Witt et al. 2006; Malloci et al. 2007a). As ionized PAHs are attractive candidates for the wellknown but enigmatic diffuse interstellar bands, laboratory studies have also focused on recording electronic spectra of PAH cations (Salama et al. 2011; Steglich et al. 2011). The charge state of interstellar PAHs is of wider interest. UV photons can also lead to destruction of interstellar PAHs (Le Page et al. 2001; Berné \& Tielens 2012). Experiments have explored this fragmentation process (Jochims et al. 1994, 1996; Ekern et al. 1998; Zhen et al. 2014a, 2014b) and the IR characteristics of the resulting species have been quantum chemically calculated (e.g., Bauschlicher \& Ricca 2013; Mackie et al. 2015). Finally, detailed models have been constructed to assess their lifetime under different interstellar conditions (Le Page et al. 2001; Montillaud et al. 2013).

It is clear that interstellar PAHs are expected to be greatly influenced by the strong radiation field in space through ionization and fragmentation processes (Leach 1986; Verstraete et al. 1990; Bakes \& Tielens 1994; Le Page et al. 2001; Berné \& Tielens 2012; Montillaud et al. 2013). However, until now, experimental studies have focused either on the ionization or the fragmentation aspects (Jochims et al. 1994, 1996, 1997; Ekern et al. 1998; Zhen et al. 2014a, 2014b). In fact, these two processes will be in competition and both aspects have to be considered simultaneously. In this Letter, we perform a systematic study in the $8-40 \mathrm{eV}$ range of the photo-chemical behavior of three PAH cations that sample relevant sizes of interstellar PAHs using the SOLEIL synchrotron facility. The experiments are described in Section 2, the results are analyzed in Section 3, and discussed in Section 4.

\section{EXPERIMENTAL METHODS}

We have studied the photo-chemical processes of $\mathrm{PAH}$ cations in the $8-40 \mathrm{eV}$ range using $\mathrm{i}-\mathrm{PoP}$, our instrument for Photodissociation of PAHs connected to a vacuum ultraviolet (VUV) synchrotron beamline. i-PoP is described in detail in Zhen et al. (2014a). Briefly, PAHs are sublimated in an oven at an appropriate temperature, ionized by an electron gun (Egun), and then transported into a quadrupole ion-trap (QIT) via an ion gate. Once the ions are trapped, external electrical waveforms (SWIFT) are applied to the end cap electrodes to 


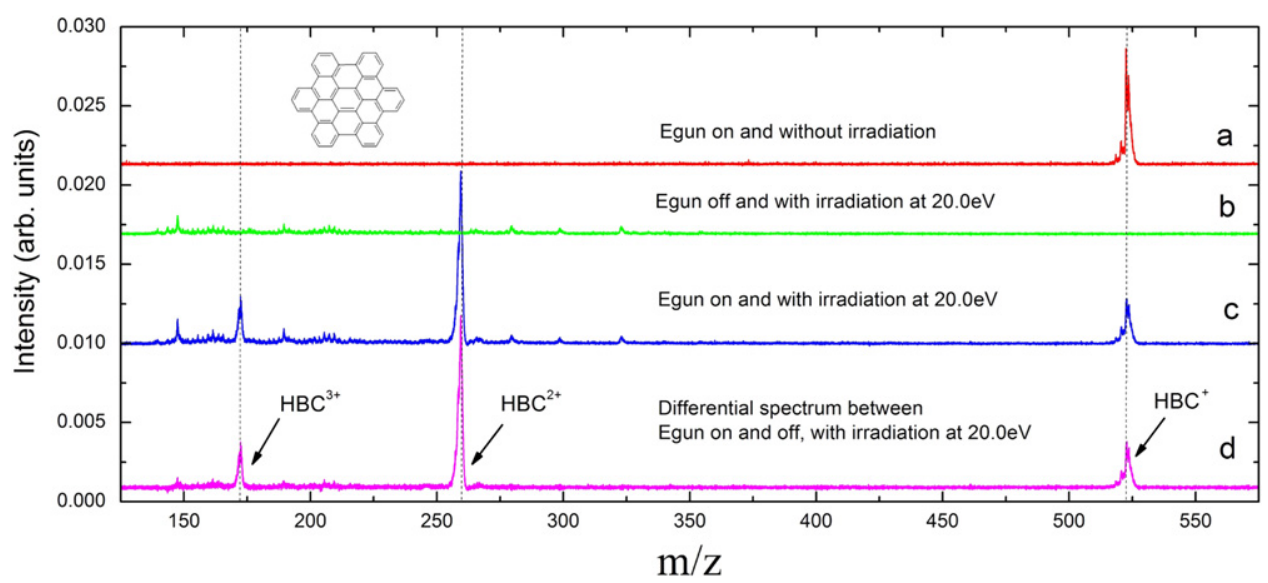

Figure 1. Mass spectrum of the products resulting from photo-irradiation of $\mathrm{HBC}$ cations at $20.0 \mathrm{eV}(62.0 \mathrm{~nm})$. (a) Egun on and without irradiation. (b) Egun off and with irradiation. (c) Egun on and with irradiation for $500 \mathrm{~ms}$. (d) Differential mass spectrum between experiments (c) and (b).

isolate a specific range of mass/charge $(\mathrm{m} / \mathrm{z})$ species (Doroshenko \& Cotter 1996). After a short time delay, the ion cloud thermalizes to room temperature ( 298 K) through collisions with He buffer gas. The cations are then irradiated by synchrotron radiation. The ion-trap content is subsequently released and analyzed using a reflectron time-of-flight mass spectrometer. A LABVIEW program automates the full data acquisition process.

The i-PoP setup is connected to the undulator-based VUV beamline DESIRS at the SOLEIL synchrotron facility in SaintAubin, France (Nahon et al. 2012). The DESIRS beamline is well suited for PAH cation studies because of its high photon flux, its broad and easy tunability in the $5-40 \mathrm{eV}$ range, as well as its highly focused beam. In order to maximize the photon flux on our very diluted targets, we used the $6.65 \mathrm{~m}$ normal incidence monochromator at the zeroth order, leading to a typical photon flux in the $10^{14}-10^{15}$ photon $\mathrm{s}^{-1}$ range in a $7 \%$ bandwidth. Another crucial feature of the beamline, in the context of mass spectrometry, lies in its spectral purity, i.e., the absence of any high harmonics of the undulator which are very efficiently filtered-out by a gas filter up to $16 \mathrm{eV}$ (Argon) and by the cutoff of the grating coatings above $21 \mathrm{eV}$. Absolute incident photon fluxes are measured using calibrated photodiodes. The radiation beam is sent directly into the QIT, with a spot size of $\simeq 1.5 \mathrm{~mm}^{2}$ in the center position. To achieve this, an additional chamber is connected with the differential pumping stage that accommodates the pressure difference between the i-PoP chamber $\left(10^{-7}\right.$ mbar) and the beamline port $\left(10^{-8} \mathrm{mbar}\right)$. The new chamber includes a retractable beam shutter (XRS2, Vincent Associates) that provides a minimum open time $(\simeq 1 \mathrm{~ms})$ under high-vacuum conditions. The beam shutter is externally triggered to guarantee that the ion cloud is irradiated for a specified amount of time during each scan cycle.

\section{RESULTS}

We have studied the photo-chemical processing of three PAH cations: coronene $\left(\mathrm{C}_{24} \mathrm{H}_{12}{ }^{+} ; \mathrm{m} / z=300.09\right)$, ovalene $\left(\mathrm{C}_{32} \mathrm{H}_{14}{ }^{+} ; \mathrm{m} / z=398.11\right)$, and hexa-peri-hexabenzocoronene $\left(\mathrm{C}_{42} \mathrm{H}_{18}{ }^{+} ; \mathrm{HBC}^{+} ; m / z=522.14\right)$. These particular PAHs are selected for their potential astrophysical interest (Tielens 2005), their commercial availability, their easy handling and, above all, because they span an astrophysically relevant range

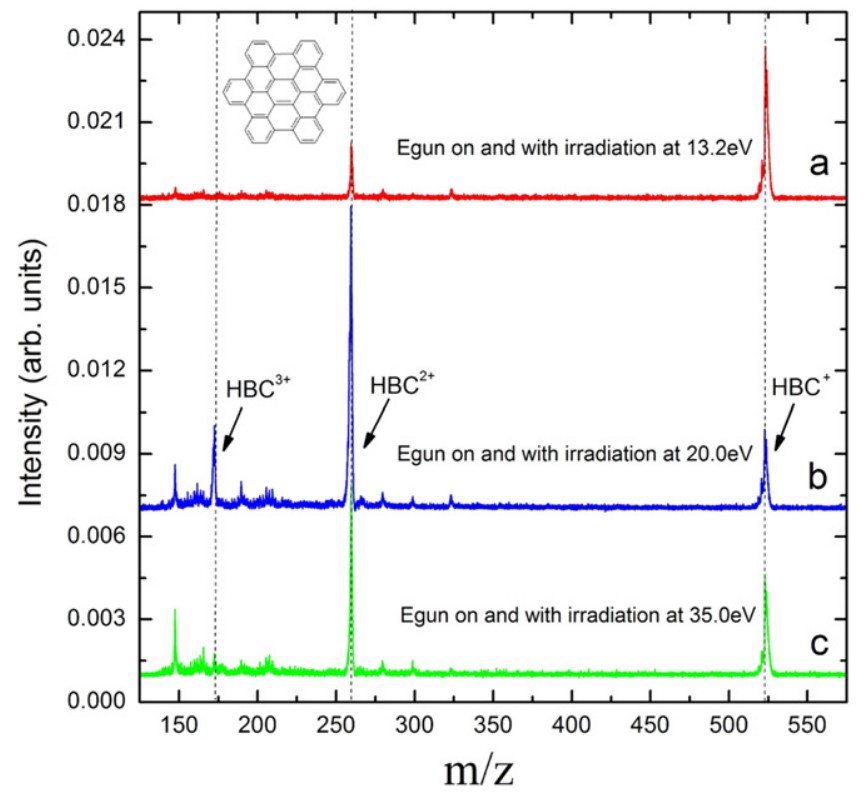

Figure 2. Mass spectrum of the products resulting from photo-irradiation of $\mathrm{HBC}$ cations at different energies for $500 \mathrm{~ms}$ : (a) $13.2 \mathrm{eV}(93.9 \mathrm{~nm})$, (b) $20.0 \mathrm{eV}(62.0 \mathrm{~nm})$, and $(\mathrm{c}) 35.0 \mathrm{eV}(35.4 \mathrm{~nm})$.

of sizes. The experimental results obtained with i-PoP are summarized in Figures 1-4 and Table 1.

Figure 1 summarizes the results for the $\mathrm{HBC}$ cation at $20.0 \mathrm{eV}(62.0 \mathrm{~nm})$. The mass spectrum before irradiation reveals a small amount of residual fragmentation (peaks due to $1 \mathrm{H}$ and $2 \mathrm{H}$ loss) as a byproduct of the electron impact ionization process as well as the presence of the ${ }^{13} \mathrm{C}$ isotope (blended with the main mass peak) at natural abundance. After irradiation, a low level of contamination is present when no HBC is stored (Egun is off), due to the interaction of the synchrotron beam with PAH pollutions on the walls from previous experiments (Figure 1(b)). These contaminating peaks are also present in the irradiation experiments of $\mathrm{HBC}^{+}$ (Figure $1(\mathrm{c})$ ) but can be quite well corrected for (Figure 1(d)). As the contamination peaks are sufficiently off-set from $\mathrm{HBC}^{+}$ photo-products, they do not interfere with the analysis below, and we have not explicitly corrected the mass spectra in other experiments. 


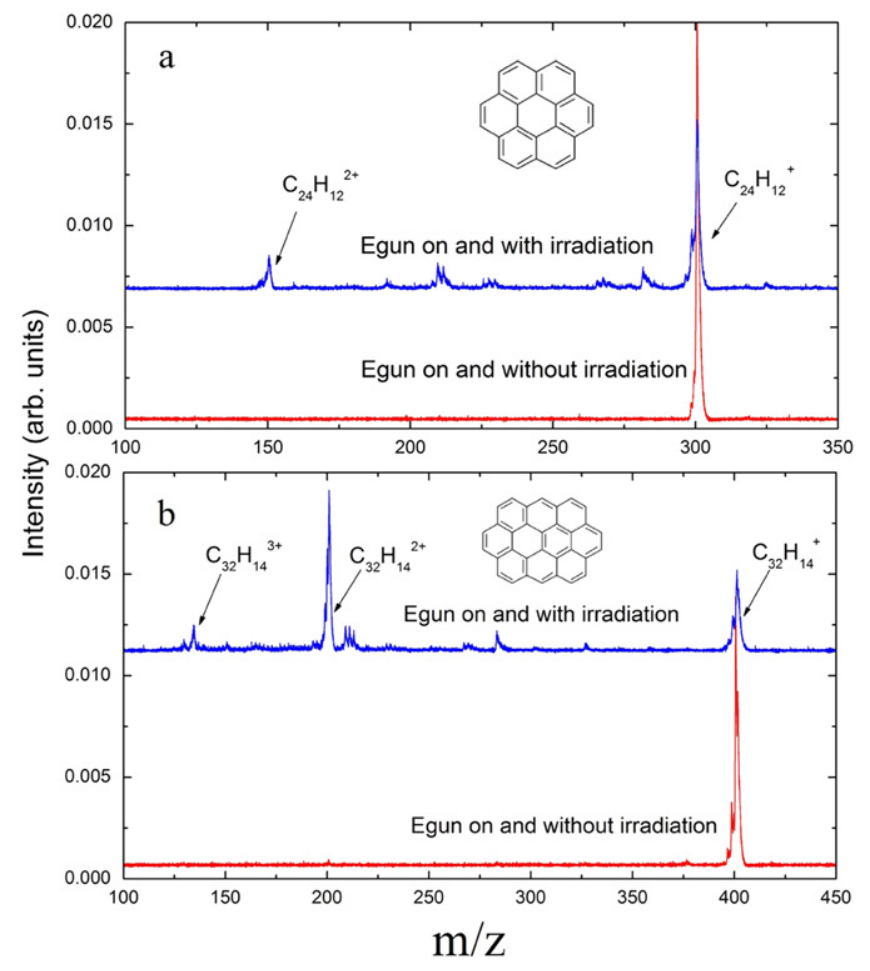

Figure 3. Panel (a): mass spectrum of Corenene cation irradiated at $20.0 \mathrm{eV}$ $(62.0 \mathrm{~nm})$ for $200 \mathrm{~ms}$, with and without irradiation. Panel (b): mass spectrum of Ovalene cation irradiated at $21.0 \mathrm{eV}(59.0 \mathrm{~nm})$ for $500 \mathrm{~ms}$, with and without irradiation.

The $\mathrm{HBC}^{+}$irradiation results at $20.0 \mathrm{eV}$ (Figure $1(\mathrm{~d})$ ) show strong peaks due to the $\mathrm{HBC}$ dication $\left(\mathrm{C}_{42} \mathrm{H}_{18}{ }^{2+} ; \mathrm{HBC}^{2+}\right.$; $m / z=261.07)$ and trication $\left(\mathrm{C}_{42} \mathrm{H}_{18}{ }^{3+} ; \mathrm{HBC}^{3+} ; \mathrm{m} / z=174.05\right)$ but little evidence for loss of hydrogen (beyond that due to the effects of the Egun; see below). Similar results are obtained at $35.0 \mathrm{eV}$ (Figure 2(c)), albeit that the overall photo-processing is less intense, reflecting the lower absorption cross section of HBC cation at this wavelength $(35.4 \mathrm{~nm})$ compared to $62.0 \mathrm{~nm}$ (Malloci et al. 2007a). At low photon energies $(13.2 \mathrm{eV}$; $93.9 \mathrm{~nm}$; Figure 2(a)), only the $\mathrm{HBC}$ dication is present. Figure 2(b) is identical to Figure 1(c) and is shown for ease of comparison for three different irradiation energies. Irradiation of ovalene shows grossly the same behavior with production of ovalene di- and tri-cations $\left(\mathrm{C}_{32} \mathrm{H}_{14}{ }^{2+} ; \mathrm{m} / z=199.05\right.$ and $\mathrm{C}_{32} \mathrm{H}$ $\left.14^{3+} ; m / z=132.67\right)$, as illustrated in Figure 3(b). We have selected to show ovalene at $21 \mathrm{eV}$ with $500 \mathrm{~ms}$ irradiation to bring out the small amount of $\mathrm{C}_{32} \mathrm{H}_{14}{ }^{3+}$ formation at these conditions. For coronene, the dication $\left(\mathrm{C}_{24} \mathrm{H}_{12}{ }^{2+}\right.$; $m / z=150.04)$ is produced but the presence of the trication could not be confirmed as it is outside the QIT mass spectrometer's range (Figure 3(a); March 1997). Appearance energies for the different ion states are summarized in Table 1 and compared to measured, calculated, or estimated ionization potentials. We note that new ionization states become apparent immediately when the photon energy exceeds the ionization potential to the next ionization state. Hence, the appearance of the trication reflects the importance of (sequential) two photon processes. The importance of two photon processes is in agreement with estimates of the mean number of photons absorbed for these PAHs, given the large absorption cross section calculated by Malloci et al. (2007a).

The fragmentation and ionization behavior of coronene, ovalene, and HBC cations at about 13.2 and $20 \mathrm{eV}$ are illustrated in Figure 4. We note that for a given photon energy, the ionization yield increases with PAH size, i.e., from right to left, while the loss of $\mathrm{H}$ is more important for small PAHs. After $20 \mathrm{eV}$ irradiation, the coronene and ovalene cations show clear evidence for the loss of two and four hydrogen atoms. We also note photo-fragments of the coronene and ovalene dications. With increasing energy, i.e., comparing the lower and corresponding upper panels, ionization yield and fragmentation yield increase but the same pattern remains: ionization completely dominates for $\mathrm{HBC}$ while fragmentation can compete with ionization for coronene. We can compare this quantitatively at high energies where ionization and fragmentation are expected to completely dominate over IR fluorescence. For fragmentation and ionization, we have ignored the small correction for the two photon process. We have corrected the strength of fragment peaks in the spectra for the background by subtracting a fraction of the e-gun fragmentation. To determine this fraction, we assume that fragments produced by the e-gun will be ionized at the same way as the parent and isotopic species. Hence, we ignore the effect of small shift in ionization potential expected for the fragments (Malloci et al. 2008). With this assumption, the fraction is then determined by scaling the strength of the parent plus isotope in the spectrum by the parent plus isotope in the e-gun spectrum. In this way, we estimate that -at $20 \mathrm{eV}$ - only around $\sim 3 \%$ of the HBC cations fragment while $\sim 97 \%$ are ionized. For ovalene and coronene, the ionization yields are $70 \% \pm 10 \%$ and $25 \% \pm 3 \%$, respectively (e.g., fragmentation yields of $30 \% \pm 10 \%$ and $75 \% \pm 3 \%)$. Details of the procedures will be reported in a forthcoming paper (P. Castellanos et al. 2015, in preparation).

\section{DISCUSSION AND ASTRONOMICAL IMPLICATIONS.}

At the high photon energies of our experiments, several processes-including ionization and fragmentation-can compete in the relaxation process. Calculated UV absorption cross sections for neutral and ionized PAHs reveal broad, blended peaks due to $\pi^{\star} \leftarrow \pi, \pi^{\star} \leftarrow \sigma, \quad \sigma^{\star} \leftarrow \pi$, and $\sigma^{\star} \leftarrow \sigma$ transitions, involving superexcited (bound) states, which lie above the ionization continuum (Malloci et al. 2007a). Apart from these single electron transitions, valence shell twoelectron processes in which more than one electron is involved, may also be important (Becker \& Shirley 1996), particularly at higher energies $(\gtrsim 16 \mathrm{eV}$; A. Marciniak et al. 2015, in preparation). Due to the high electron density of states, coupling is very fast among these states, and highly excited PAHs can rapidly (tens of femtoseconds; A. Marciniak et al. 2015, in preparation) relax to lower lying states-through conical intersections-including the ionizing continuum. If no ionization occurs, this internal conversion will leave the species in a highly vibrationally excited state in the ground electronic state. Internal vibrational relaxation (IVR) will then occur, sharing the vibrational energy among all the modes on a timescale of tens of nanoseconds. The energy will eventually be radiated away in the IR through vibrational transitions on a timescale of $\sim 1 \mathrm{~s}$, but if the excitation is sufficiently high, enough energy can accumulate in one vibrational mode corresponding to a given $\mathrm{C}-\mathrm{H}$ bond, leading to the unimolecular fragmentation of the cation in the electronic ground 

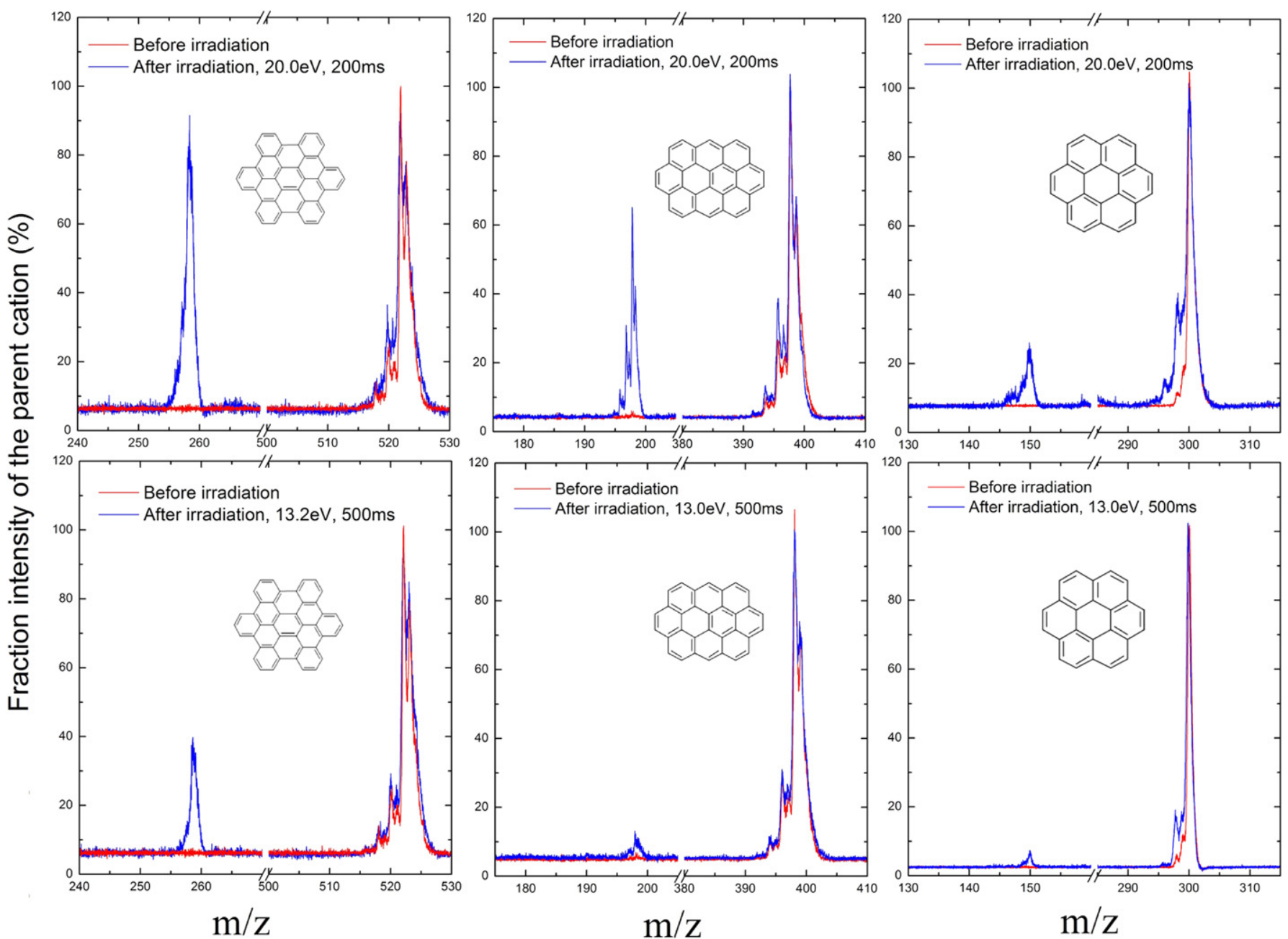

Figure 4. Normalized intensity of PAH cations before and after irradiation. Top row: irradiation for $200 \mathrm{~ms}$ at $20.0 \mathrm{eV}(62.0 \mathrm{~nm})$. Bottom row: irradiation for $500 \mathrm{~ms}$ at $\simeq 13.2 \mathrm{eV}(93.9 \mathrm{~nm})$. From left to right in each row, HBC, ovalene, and coronene cation.

Table 1

Appearance Energies and Ionization Potentials for Doubly and Triply Charged PAH Parent Ions

\begin{tabular}{|c|c|c|c|c|c|}
\hline \multirow{2}{*}{ Name } & \multirow{2}{*}{ Formula } & \multicolumn{2}{|c|}{ Ionization Potential $^{\mathrm{a}}$} & \multicolumn{2}{|c|}{ Appearance Potential ${ }^{\mathrm{a}}$} \\
\hline & & $\mathrm{PAH}^{+} \rightarrow \mathrm{PAH}^{2+}$ & $\mathrm{PAH}^{2+} \rightarrow \mathrm{PAH}^{3+}$ & $\mathrm{PAH}^{+} \rightarrow \mathrm{PAH}^{2+}$ & $\mathrm{PAH}^{2+} \rightarrow \mathrm{PAH}^{3+}$ \\
\hline $\begin{array}{l}\text { Hexa-peri- } \\
(\mathrm{HBC})\end{array}$ & $\mathrm{C}_{42} \mathrm{H}_{18}{ }^{+}$ & $9.0^{\mathrm{e}}$ & $13.0^{\mathrm{c}}$ & $\leqslant 8.5 \pm 0.6^{\mathrm{f}}$ & $16.5 \pm 2.0$ \\
\hline Ovalene & $\mathrm{C}_{32} \mathrm{H}_{14}{ }^{+}$ & $9.6^{\mathrm{e}}$ & $15.4^{c}$ & $9.4 \pm 0.6$ & $18.0 \pm 2.0$ \\
\hline Coronene & $\mathrm{C}_{24} \mathrm{H}_{12}{ }^{+}$ & $11.3^{\mathrm{b}}$ & $17.2^{\mathrm{c}}$ & $10.5 \pm 0.7$ & $\ldots^{\mathrm{d}}$ \\
\hline
\end{tabular}

${ }^{\mathrm{a}} \mathrm{In} \mathrm{eV}$.

b (Tobita et al. 1994).

${ }^{\mathrm{c}}$ Estimated from circular disk approximation (Tielens 2005).

${ }^{\mathrm{d}}$ Outside of the QIT mass spectrometer range.

e (Malloci et al. 2007b).

${ }^{\mathrm{f}}$ The HBC dication is already present at the lowest energy in our experiments.

state. Ionization competes, thus, with Internal Conversion, which is then followed by IVR, and then fragmentation or IR emission. Experiments on small, neutral PAHs (up to coronene) have shown that the ionization yield increases with increased photon energy and typically reaches unity some $9 \mathrm{eV}$ above the ionization potential (Jochims et al. 1996, 1997). For coronene cations, experiments show that fragmentation $(\mathrm{H}$ loss) occurs on a timescale $10^{-4} \mathrm{~s}$ for an internal energy of $\simeq 12$
eV (Jochims et al. 1994). In the ISM, fragmentation can occur on longer timescales at which fragmentation is in competition with IR cooling. This happens for coronene cation at an internal energy of $\sim 9 \mathrm{eV}$ (Montillaud et al. 2013).

In the analysis of the experimental results presented in Section 3, several points have to be kept in mind. First, for all PAHs, the UV absorption cross section per C-atom is very similar in strength and in general behavior with energy; e.g., 
the absolute cross section is almost a factor of two larger for HBC (42 C-atoms) than for coronene (24 C-atoms). Second, the UV absorption cross section typically increases with photon energy by a factor 10 from $\simeq 8 \mathrm{eV}$ to a broad $(\simeq 2 \mathrm{eV})$ maximum around $\simeq 16 \mathrm{eV}$ and then decreases again by a factor 3 to $25 \mathrm{eV}$ (Malloci et al. 2007a). Third, the synchrotron beam may not overlap with the ion cloud in the same way for the different species. All these points imply that we should only consider the relative importance of the different processes when comparing different species or when comparing measurements for the same species at different energies.

The experimental results reveal that ionization competes well with fragmentation for PAHs cations as small as coronene but for PAHs as large as HBC ionization dominates. We consider that two effects play a role. First, the larger number of electrons lead to a higher density of electronic states. Hence, the electronically excited species have to traverse more "electronic" phase space and drop through more conical intersections before reaching the ground electronic state. The associated longer "electronic" lifetime facilitates transfer to the ionizing continuum and will lead to a relatively higher ionization yield. Second, a larger species will need a higher internal energy to reach the same level of excitation because of the higher number of vibrational modes into which the energy can be distributed. Specifically, for HBC, the internal energy has to be almost a factor of two higher than for coronene for the same excitation level of the vibrational modes. Hence, the fragmentation yield will rapidly decrease when going from coronene to HBC (P. Castellanos et al. 2015, in preparation). While we have focused here on the behavior of $\mathrm{HBC}^{+}$, we note that $\mathrm{HBC}^{2+}$ shows a similar behavior with little apparent fragmentation compared to ionization over the relevant energy range.

Our results imply that large $\mathrm{PAH}$ cations are inherently photo-stable in the ISM. Fragmentation will become important when only electronic states below the next ionization potential of the species are accessible, otherwise photoionization will quench the competing photodissociation process. For HBC in $\mathrm{HI}$ region (with $h \nu \leqslant 13.6 \mathrm{eV}$ ), this corresponds to $\mathrm{HBC}^{2+}$. For larger species such as circumcoronene $\left(\mathrm{C}_{54} \mathrm{H}_{18}\right)$ even the trication is accessible with photons less than $13.6 \mathrm{eV}$. Fragmentation is then not expected to become important until the ionization parameter, $\gamma\left(=G_{o} T^{1 / 2} / n_{e}\right.$ with $G_{o}$ the strength of the radiation field in units of the Habing field, $T$ the gas temperature, and $n_{e}$ the electron density), exceeds $\simeq 10^{6}$ (Tielens 2005) and, thus, only close to a bright star. Some fragmentation of $\mathrm{HBC}^{+}$may, in principle, occur when $\mathrm{HBC}^{2+}$ recombines. However, recombination limits the internal excitation energy of $\mathrm{HBC}^{+}$to the ionization potential $(9 \mathrm{eV}$ for $\mathrm{HBC}^{+}$) and little fragmentation will occur at such low energies for large species (Montillaud et al. 2013; P. Castellanos et al. 2015, in preparation).

In the reflection nebula, NGC 7023, observations have revealed that PAHs are rapidly destroyed near the illuminating star while the abundance of the fullerene, $\mathrm{C}_{60}$ increases (Berné \& Tielens 2012). A similar behavior is observed in other regions in space (Castellanos et al. 2014). This has been interpreted as the result of photochemical evolution leading to loss of $\mathrm{H}$-atoms, the formation of graphene sheets, followed by a curling up of these sheets into cages and then fullerenes (Berné \& Tielens 2012; Montillaud et al. 2013; Berné et al. 2015). There is good experimental support for the process of $\mathrm{H}$-loss and the formation of pure carbon sheets, clusters, or cages (Ekern et al. 1998; Zhen et al. 2014a; West et al. 2015). Recently, we have also obtained experimental support for the formation of fullerenes through this process (Zhen et al. 2014b). These latter studies were specifically designed to avoid the ionization versus fragmentation competition by pumping the molecules through the sequential events of multi-photon absorptions all below the ionization potential. In conjunction with the study reported here, we infer that H-loss, graphene formation, and cage and fullerene formation will only occur when ionization is a marginal process. As these experiments demonstrate, multi-photon events are one, very relevant scenario and the theoretical study by Montillaud et al. (2013) has already pointed out the importance of multi-photon events in the fragmention of interstellar PAHs in neutral hydrogen regions in the ISM. These absorption events should then involve photons below the ionization potential of the species involved. Alternatively, in $\mathrm{H}$ II regions, much higher energy photons are available and, in principle, ionization and fragmentation by a single photon may become possible. However, we note that only a small amount $(\leqslant 2 \%)$ of fragmentation of $\mathrm{HBC}^{+}$even for photon energies as high as $35.0 \mathrm{eV}$, some $26.0 \mathrm{eV}$ above the ionization potential.

\section{CONCLUSION}

Photo-chemical processing with synchrotron irradiation of $\mathrm{PAH}$ cations in the range of $8-40 \mathrm{eV}$ reveals that ionization becomes more and more important when the size of the PAH increases, quenching the competing photodissociation process. For PAHs of some $50 \mathrm{C}$-atoms, ionization fully dominates over fragmentation. Hence, in addition to the kinetic parameters involved in the fragmentation process, the ionization process has to be quantified for a proper assessment of the stability of PAHs in space. In other words, we consider it likely that variations in the ionization characteristics of PAHs are as important in evaluating the composition of the interstellar PAH family as the kinetic fragmentation parameters. The ionization state may well leave its imprint on the IR spectrum of PAHs (Bakes et al. 2001a; Malloci et al. 2007a) and, for example, ionized large PAHs have been proposed as the carriers of a characteristic band at $7.90 \mu \mathrm{m}$ prominent in some planetary nebula (Joblin et al. 2008). Further observational studiessupported by experimental or quantum chemical studies-are warranted.

We are grateful to M. J. A. Witlox and R. Koehler for technical support. Studies of interstellar chemistry at Leiden Observatory are supported through advanced-ERC grant 246976 from the European Research Council, through a grant by the Dutch Science Agency, NWO, as part of the Dutch Astrochemistry Network, and through the Spinoza premie from the Dutch Science Agency, NWO. We also acknowledge support from the European Research Council under the European Union's Seventh Framework Programme ERC2013-SyG, grant agreement No. 610256 NANOCOSMOS, as well as from the EU Transnational Access Program CALYPSO. We are indebted to J.-F. Gil for technical help in installing the i-PoP set-up onto the DESIRS beamline and to the general staff of SOLEIL for running the facility under project No. 20130911. 


\section{REFERENCES}

Allamandola, L. J., Tielens, A. G. G. M., \& Barker, J. R. 1989, ApJS, 71, 733

Armus, L., Charmandaris, V., Bernard-Salas, J., et al. 2007, ApJ, 656, 148

Bakes, E. L. O., \& Tielens, A. G. G. M. 1994, ApJ, 427, 822

Bakes, E. L. O., Tielens, A. G. G. M., \& Bauschlicher, C. W. 2001a, ApJ, 556, 501

Bakes, E. L. O., Tielens, A. G. G. M., Bauschlicher, C. W., Hudgins, D. M., \& Allamandola, L. J. 2001b, ApJ, 560, 261

Bauschlicher, C. W., Jr., \& Ricca, A. 2013, ApJ, 776, 102

Becker, U., \& Shirley, D. A. (ed.) 1996, in VUV and Soft X-Ray Photoionization (Berlin: Springer)

Berné, O., \& Tielens, A. G. G. M. 2012, PNAS, 109, 401

Berné, O., Montillaud, J., \& Joblin, C. 2015, A\&A, in press

Castellanos, P., Berné, O., Sheffer, Y., Wolfire, M. G., \& Tielens, A. G. G. M. 2014, ApJ, 794, 83

Doroshenko, M. V., \& Cotter, R. J. 1996, RCMS, 10, 65

Ekern, S. P., Marshall, A. G., Szczepanski, J., \& Vala, M. 1998, JPCA, 102,3498

Genzel, R., Lutz, D., Sturm, E., et al. 1998, ApJ, 498, 579

Joblin, C., Szczerba, R., Berné, O., \& Szyszka, C. 2008, A\&A, 490, 189

Jochims, H. W., Baumgärtel, H., \& Leach, S. 1996, A\&A, 314, 1003

Jochims, H. W., Rühl, E., Baumgärtel, H., Tobita, S., \& Leach, S. 1994, ApJ, 420, 307

Jochims, H. W., Rühl, E., Baumgärtel, H., Tobita, S., \& Leach, S. 1997, IJMSI, $167 / 168,35$

Leach, S. 1986, JESRP, 41, 427

Le Page, V., Snow, T. P., \& Bierbaum, V. M. 2001, ApJS, 132, 233
Lutz, D., Sturm, E., Tacconi, L. J., et al. 2007, ApJL, 661, L25

Mackie, C. J., Peeters, E., Bauschlicher, C. W., Jr., \& Cami, J. 2015, ApJ, 799, 131

Malloci, G., Joblin, C., \& Mulas, G. 2007a, A\&A, 462, 627

Malloci, G., Joblin, C., \& Mulas, G. 2007b, CP, 332, 353

Malloci, G., Mulas, G., Cecchi-Pestellini, C., \& Joblin, C. 2008, A\&A, 489, 1183

March, R. E. 1997, JMS, 32, 351

Montillaud, J., Joblin, C., \& Toublanc, D. 2013, A\&A, 552, A15

Nahon, L., de Oliveira, N., Garcia, G. A., et al. 2012, J. Synchrotron Rad., 19, 508

Puget, J. L., \& Leger, A. 1989, ARA\&A, 27, 161

Salama, F., Galazutdinov, G. A., Krełowski, J., et al. 2011, ApJ, 728, 154

Sellgren, K. 1984, ApJ, 277, 623

Steglich, M., Bouwman, J., Huisken, F., \& Henning, T. 2011, ApJ, 742, 2

Tielens, A. G. G. M. 2005, The Physics and Chemistry of the Interstellar Medium (1st ed.; Cambridge: Cambridge Univ. Press)

Tielens, A. G. G. M. 2008, ARA\&A, 46, 289

Tielens, A. G. G. M. 2013, RvMP, 85, 1021

Tobita, S., Leach, S., Jochins, H. W., et al. 1994, CaJPh, 72, 1060

Verstraete, L., Leger, A., d'Hendecourt, L., Defourneau, D., \& Dutuit, O. 1990, A\&A, 237, 436

West, B., Us-li Bacchitta, F., Sabbah, H., et al. 2015, JPCA, 119, 1127

Witt, A. N., Gordon, K. D., Vijh, U. P., et al. 2006, ApJ, 636, 303

Zhen, J., Castellanos, P., Paardekooper, D. M., Linnartz, H., \& Tielens, A. G. G. M. 2014b, ApJL, 797, L30

Zhen, J., Paardekooper, D. M., Candian, A., Linnartz, H., \& Tielens, A. G. G. M. 2014a, CPL, 592, 211 